\title{
Thin Lehman Matrices and Their Graphs
}

\author{
Jonathan Wang \\ Department of Mathematics \\ Harvard University, Cambridge, MA 02138, USA \\ jpwang@fas.harvard.edu
}

Submitted: Apr 24, 2010; Accepted: Nov 22, 2010; Published: XXDec 3, 2010

Mathematics Subject Classification: 05B20

\begin{abstract}
Two square 0,1 matrices $A, B$ are a pair of Lehman matrices if $A B^{T}=J+d I$, where $J$ is the matrix of all 1 s and $d$ is a positive integer. It is known that there are infinitely many such matrices when $d=1$, and these matrices are called thin Lehman matrices. An induced subgraph of the Johnson graph may be defined given any Lehman matrix, where the vertices of the graph correspond to rows of the matrix. These graphs are used to study thin Lehman matrices. We show that any connected component of such a graph determines the corresponding rows of the matrix up to permutations of the columns. We also provide a sharp bound on the maximum clique size of such graphs and give a complete classification of Lehman matrices whose graphs have at most two connected components. Some constraints on when a circulant matrix can be Lehman are also provided. Many general classes of thin Lehman matrices are constructed in the paper.
\end{abstract}

\section{Introduction}

Lehman matrices were defined by Lütolf and Margot [7] to aid in the classification of minimally nonideal matrices, which are a key tool for understanding when the set covering problem can be solved using linear programming (we refer the reader to [2] for more information on minimally nonideal matrices). Lehman matrices lie at the heart of Lehman's central theorem on minimally nonideal matrices $[5,6]$. He showed that for $m \geq n$ almost every $m \times n$ minimally nonideal matrix contains a unique $n \times n$ Lehman matrix. Bridges and Ryser [1] showed that every Lehman matrix is $r$-regular for some integer $r \geq 2$, i.e., each row and column sums to $r$. Two infinite families of Lehman matrices are known: the point-line incidence matrices of finite nondegenerate projective planes, a widely studied topic [4], and thin Lehman matrices. Thin Lehman matrices were defined and studied by Cornuéjols et al. [3]. 
Two square $n \times n$ matrices $A, B$ form a pair of Lehman matrices if each matrix has only 0,1 as entries, and $A B^{T}=J+d I$ for some positive integer $d$ (where $J$ is the matrix of all ones). Lütolf and Margot enumerated all Lehman matrices with $n \leq 11$. If $A=B$, then $A A^{T}=J+d I$, and $A$ is by definition the point-line incidence matrix of a nondegenerate projective plane of order $d$. The classification of finite nondegenerate projective planes is an open problem, and the only known orders are prime powers [4]. A Lehman matrix is called thin in the case $d=1$. A matrix is circulant if each row is a right 1-cyclic shift of the previous row. Given integers $r, s \geq 2$ and $n=r s-1$, let the circulant matrix $C_{n}^{r}$ be the $n \times n$ matrix with columns indexed by $\mathbb{Z} / n \mathbb{Z}$ and its $i$ th row equal to the incidence vector of $\{i, i+1, \ldots, i+r-1\}$, i.e., the 0,1 vector that has 1 s in the specified columns, for $i \in \mathbb{Z} / n \mathbb{Z}$. Also define the $n \times n$ circulant matrix $D_{n}^{s}$ in the same way except with rows equal to the incidence vectors of $\{i, i+r-1, i+2 r-1, \ldots, i+(s-1) r-1\}$. Cornuéjols et al. [3] noted that $C_{n}^{r}, D_{n}^{s}$ form a thin Lehman pair, which shows that there are infinitely many thin Lehman matrices. Given a Lehman matrix $A$, Cornuéjols et al. introduced a graph $G_{A}$, which we call the Johnson subgraph induced by $A$, to study properties of the matrix $A$. The graph $G_{A}$ has the rows of $A$ as vertices, and two rows are adjacent if each row has all but one 1 in the same column as the other row.

In this paper, we continue the study of thin Lehman matrices. We investigate the Johnson subgraphs associated to thin Lehman matrices, which have particularly simple structures. In Section 3, we show that the structures of a Lehman matrix and its graph are closely related. Our main result shows that any connected component of the graph $G_{A}$ determines the corresponding rows of $A$ up to permutations of the columns. Bounds on the maximum clique size and maximum degree of a Johnson subgraph of a thin Lehman matrix are given in Section 4. We also prove that some of the bounds given are sharp. We believe the new restrictions we impose on thin Lehman matrices will make it easier to enumerate them. In Section 5, all Lehman matrices with graphs containing at most two connected components are classified. Lastly, the induced Johnson subgraph is used to provide constraints on when a circulant matrix is Lehman in Section 6. A complete classification of all Lehman matrices is, however, still lacking. A Lehman matrix may not be determined by its graph once the graph has more than two connected components, which reveals one limitation of the induced Johnson subgraph.

\section{Preliminaries}

A 0,1 matrix is $r$-regular if every row and column has exactly $r$ ones. We restate the theorem of Bridges and Ryser [1] on regularity of Lehman matrices.

Theorem 2.1 ([1], Theorem 1.2). Let $A, B$ be a Lehman pair. Then there exist integers $r, s \geq 2$ such that $A$ is $r$-regular, $B$ is s-regular, and $r s=n+d$. Moreover, $B^{T} A=$ $A B^{T}=J+d I$.

Throughout this paper, we will use $A, B$ to denote a Lehman pair of $n \times n$ matrices with $A B^{T}=J+d I$, where $A$ is $r$-regular, $B$ is $s$-regular, and $r s=n+d$. Observe that

$$
A\left(B^{T}-\frac{1}{r} J\right)=d I \Longrightarrow B^{T}=d A^{-1}+\frac{1}{r} J,
$$

THE ELECTRONiC JouRnal of COMBINATORICS 17 (2010), \#R165 
which shows that $d$ and $B$ are unique given $A$, since $B$ must be 0,1 . The matrix $B$ is called the Lehman dual of $A$. We also see that $A$ and $B$ are invertible.

Two matrices $A_{1}$ and $A_{2}$ are isomorphic, denoted $A_{1} \simeq A_{2}$, if one can be obtained from the other by permutations of rows and/or columns. Equivalently, there exist permutation matrices $P$ and $Q$ such that $P A_{1} Q=A_{2}$. If $A_{1} B_{1}^{T}=J+d I$, then

$$
\left(P A_{1} Q\right)\left(P B_{1} Q\right)^{T}=P A_{1} Q Q^{T} B_{1}^{T} P^{T}=P(J+d I) P^{T}=J+d I,
$$

so $A_{2}$ is also a Lehman matrix.

Cornuéjols et al. [3] noted that if an $n \times n$ Lehman matrix $A$ is 2-regular, then $n \geq 3$ odd, and $A \simeq C_{n}^{2}$. Therefore, we will assume $r, s>2$ in the paper.

Let $\mathbb{Z}_{\geq 0}$ denote the set of nonnegative integers. We also define the intervals of integers $[a, b]:=\{c \in \mathbb{Z} \mid a \leq c \leq b\},[a, b):=[a, b] \backslash\{b\},(a, b]:=[a, b] \backslash\{a\},(a, b):=[a, b] \backslash\{a, b\}$, and $[a]:=[1, a]$. Unless otherwise specified, we index rows and columns of an $n \times n$ matrix by $[n]$. Since we are working with 0,1 matrices, we may identify the rows and columns of a matrix with subsets of $[n]$. Given an $n \times n 0,1$ matrix $A$ and $i \in[n]$, define

$$
\operatorname{row}_{i}(A)=\left\{j \in[n] \mid a_{i j}=1\right\} \subset[n]
$$

to be the set of column indices where row $i$ has a 1 . Define $\operatorname{col}_{i}(A)$ analogously.

We provide some important observations that will be used in later proofs.

Remark 2.2. Observe that $A B^{T}=J+d I$ is equivalent to $\left|\operatorname{row}_{i}(A) \cap \operatorname{row}_{i}(B)\right|=d+1$ and $\left|\operatorname{row}_{i}(A) \cap \operatorname{row}_{j}(B)\right|=1$ for $i \neq j$. By Theorem 2.1, $A B^{T}=J+d I$ implies $B^{T} A=J+d I$, so we also have $\left|\operatorname{col}_{i}(A) \cap \operatorname{col}_{i}(B)\right|=d+1$ and $\left|\operatorname{col}_{i}(A) \cap \operatorname{col}_{j}(B)\right|=1$ for $i \neq j$. We therefore deduce that for $i \neq j$ and any $k$,

$$
\left|\operatorname{row}_{i}(A) \cap \operatorname{row}_{j}(A) \cap \operatorname{row}_{k}(B)\right| \leq 1 .
$$

Since $A$ is invertible, the row vectors of $A$ must be linearly independent. Therefore,

$$
\operatorname{row}_{i}(A) \neq \operatorname{row}_{j}(A)
$$

for $i \neq j$. Note that (1) and (2) also hold with $A$ and $B$ switched or with rows replaced by columns.

We now define the Johnson subgraph $G_{A}$ induced by an $r$-regular 0,1 matrix $A$. The vertices $V\left(G_{A}\right)$ are the rows $[n]$ of $A$. Two rows $i$ and $j$ are adjacent in $G_{A}$ if

$$
\left|\operatorname{row}_{i}(A) \cap \operatorname{row}_{j}(A)\right|=r-1 .
$$

The vertices of the Johnson graph $J(n, r)$ are the size $r$ subsets of $[n]$, and two vertices are adjacent if their intersection has size $r-1$. Thus, $G_{A}$ is the subgraph of the Johnson graph induced by the rows of $A$. If $A$ is a Lehman matrix with $d>1$, then Remark 2.2 implies that $G_{A}$ has no edges. We will therefore mainly use the graph $G_{A}$ to study $A$ when $A$ is a thin Lehman matrix.

Example. The Johnson subgraph induced by $C_{n}^{r}$ is a single cycle with $n$ vertices. 


\section{$3 \quad$ Structure of graphs}

In this section we explore the relation between the structure of a thin Lehman matrix $A$ and the Johnson subgraph $G_{A}$. We show that the structures of interest in these graphs are paths and cliques. At the end of the section we prove the following theorem.

Theorem 3.1. Suppose $A$ is a thin Lehman matrix. Let $W \subset V\left(G_{A}\right)$ be the vertices of a connected component of $G_{A}$. Then each row $(A)$ for $i \in W$ is determined by $G_{A}$ up to permutations of the columns.

We believe that the structure of the induced Johnson subgraphs will aid in the enumeration of all nonisomorphic thin Lehman matrices.

Note that if $A_{1} \simeq A_{2}$, then $G_{A_{1}} \simeq G_{A_{2}}$ since permuting rows and columns does not affect the size of row intersections. Unfortunately, the converse does not hold. We provide a counterexample below.

Example. We give two thin Lehman matrices $A_{1}, A_{2}$ with $n=14, r=3$ such that $G_{A_{1}} \simeq G_{A_{2}} \simeq P_{1} \sqcup P_{2} \sqcup P_{3} \sqcup P_{4} \sqcup P_{4}$, where $P_{k}$ is a path with $k$ vertices. We checked with a computer program that $A_{1} \not 千 A_{2}$. In the diagram, dots represent 1 s and blank spaces represent 0s.
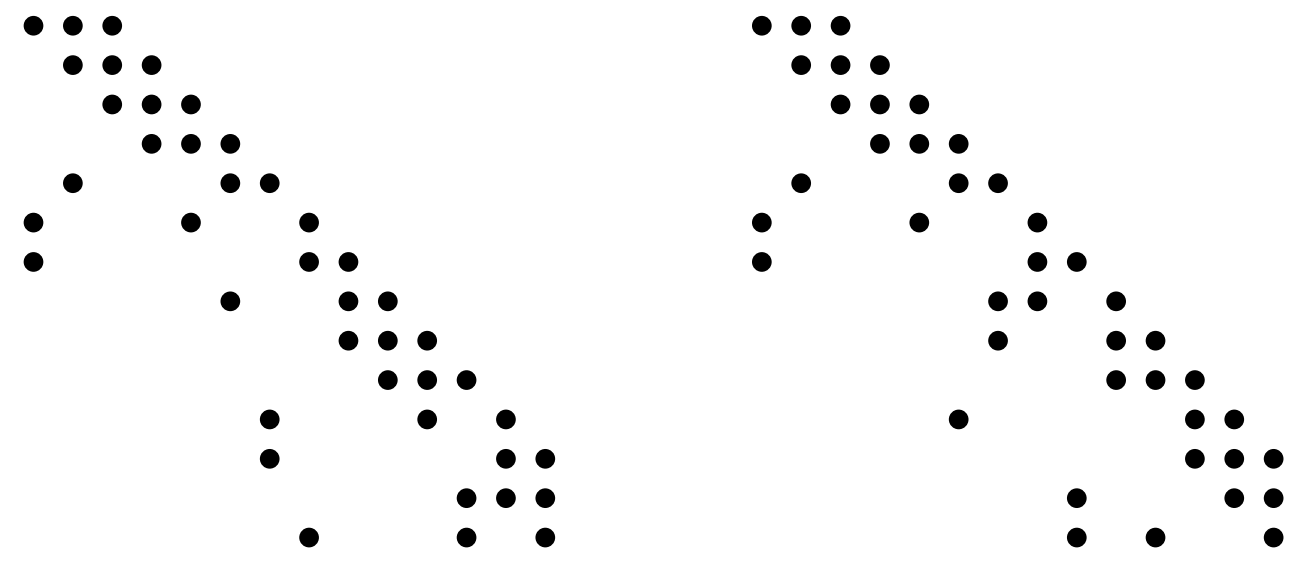

For the rest of this section, we assume $A$ is an $r$-regular thin Lehman matrix, $B$ is the $s$-regular dual, and $n=r s-1$.

\subsection{Paths}

We first build up some machinery to prove the following key lemma on the structure of the rows in $A$ corresponding to a subpath of $G_{A}$.

Lemma 3.2. Let $[k]$ be the vertices of a subpath of $G_{A}$ such that $i, i+1$ are adjacent for $i<k$, but $i, i+2$ are not adjacent for any $i<k-1$. Then either $A \simeq C_{n}^{r}$ or the columns of $A$ can be permuted such that $\operatorname{row}_{i}(A)=[i, i+r)$ for $i \in[k]$. 
If rows $[k]$ of $A$ satisfy $\operatorname{row}_{i}(A)=[i, i+r)$ for $i \in[k]$, then we say these rows have a cascading structure. We show that the cascading structure of rows in $A$ determines part of the dual matrix $B$.

Lemma 3.3. Suppose $\operatorname{row}_{i}(A)=[i, i+r)$ for $i \in[k]$. Then there exists a permutation matrix $P$ such that $\operatorname{row}_{i}(A)=\operatorname{row}_{i}(A P)$ and

$$
\operatorname{row}_{i}(B P) \cap[k+r-1]=\left\{i-r \ell, i+(r-1)+r \ell \mid \ell \in \mathbb{Z}_{\geq 0}\right\} \cap[k+r-1]
$$

for $i \in[k]$. That is, we can simultaneously permute the columns of $A$ and $B$ such that $B$ has the above form without changing the first $k$ rows of $A$.

Proof. The claim is clear for $k=1$, so assume otherwise. For $i \in[2, k)$,

$$
\operatorname{row}_{i-1}(A) \cap \operatorname{row}_{i}(A)=[i, i+r-2] \quad \text { and } \quad \operatorname{row}_{i}(A) \cap \operatorname{row}_{i+1}(A)=[i+1, i+r-1],
$$

so $\{i, i+r-1\}=\operatorname{row}_{i}(A) \cap \operatorname{row}_{i}(B)$ by (1). Since $\operatorname{row}_{1}(A) \cap \operatorname{row}_{1}(B)=[r] \cap \operatorname{row}_{1}(B)$ contains two elements and $\operatorname{row}_{1}(A) \cap \operatorname{row}_{2}(A)=[2, r]$, we must have $1 \in \operatorname{row}_{1}(B)$ by $(1)$. Similarly, $\operatorname{row}_{k-1}(A) \cap \operatorname{row}_{k}(A)=[k, k+r-2]$ implies $k+r-1 \in \operatorname{row}_{k}(B)$.

Suppose $k<r$. By assumption, $[k] \subset \operatorname{col}_{i}(A)$ for $i \in[k, r]$. The analog of (1) for columns implies that

$$
\left|[k] \cap \operatorname{col}_{j}(B)\right| \leq\left|\operatorname{col}_{k}(A) \cap \operatorname{col}_{r}(A) \cap \operatorname{col}_{j}(B)\right| \leq 1
$$

for any $j$. We have shown above that $i \in \operatorname{row}_{i}(B)$ for $i \in[1, k)$, which implies $i \in \operatorname{col}_{i}(B)$ for $i \in[1, k)$. By (3), we have $1 \notin \operatorname{col}_{i}(B)$ for $i \in[2, k)$. Therefore, $\operatorname{row}_{1}(B) \cap[1, k)=\{1\}$, so $\operatorname{row}_{1}(B)$ contains one element in $[k, r]$. Let $\operatorname{row}_{1}(B) \cap[k, r]=\{j\}$. Since $[k] \subset$ $\operatorname{col}_{j}(A) \cap \operatorname{col}_{r}(A)$, we can swap columns $j, r$ in both $A$ and $B$ to get $\{1, r\} \subset \operatorname{row}_{1}(B)$ while the first $k$ rows of $A$ stay the same. Thus, we have

$$
\{i, i+r-1\} \subset \operatorname{row}_{i}(B) \text { for } i \in[k-1]
$$

and $k+r-1 \in \operatorname{row}_{k}(B)$. Observe that $i \in \operatorname{col}_{i+r-1}(B)$ for $i \in[k]$. Now (3) implies that $k \notin$ $\operatorname{col}_{i+r-1}(B)$ for $i \in[k-1]$, or equivalently $\operatorname{row}_{k}(B) \cap[r, k+r)=\{k+r-1\}$. Hence $\operatorname{row}_{k}(B)$ contains one element in $[k, r)$. Let $\operatorname{row}_{k}(B) \cap[k, r)=\{j\}$. As $[k] \subset \operatorname{col}_{k}(A) \cap \operatorname{col}_{j}(A)$, we can swap columns $k, j$ in both $A$ and $B$ to get $\{k, k+r-1\} \subset \operatorname{row}_{k}(B)$ without changing the first $k$ rows of $A$. We conclude that

$$
\{i, i+r-1\} \subset \operatorname{row}_{i}(B) \text { for } i \in[k] .
$$

Suppose $k \geq r$. Then $[r-1]=\operatorname{col}_{r-1}(A) \cap \operatorname{col}_{r}(A)$. Since $i \in \operatorname{col}_{i}(B)$ for $i \in$ $[2, r)$, the analog of $(1)$ for columns implies that $1 \notin \operatorname{col}_{i}(B)$ for $i \in[2, r)$. Therefore, $\operatorname{row}_{1}(A) \cap \operatorname{row}_{1}(B)=\{1, r\}$. Similarly, $[k-r+2, k]=\operatorname{col}_{k}(A) \cap \operatorname{col}_{k+1}$, and $i \in \operatorname{col}_{i+r-1}(B)$ for $i \in[k-r+2, k)$ implies that $k \notin \operatorname{col}_{i+r-1}(B)$ for $i \in[k-r+2, k)$. Therefore, $\operatorname{row}_{k}(A) \cap \operatorname{row}_{k}(B)=\{k, k+r-1\}$. We deduce that (4) holds.

In both cases, (4) is true. Fix $i \in[k]$. Given $i-r \ell \in \operatorname{row}_{i}(B)$ for $\ell \in \mathbb{Z}_{\geq 0}$ such that $i-r(\ell+1)>0$, we have $\operatorname{row}_{i-r(\ell+1)+1}(A) \cap \operatorname{row}_{i}(B)=\{i-r \ell\}$. However, $\operatorname{row}_{i}(B)$ must 


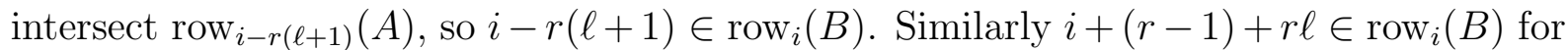
$\ell \in \mathbb{Z}_{\geq 0}$ implies $i+r-1+r(\ell+1) \in \operatorname{row}_{i}(B)$, assuming $i+(r-1)+r(\ell+1) \leq k+r-1$. Therefore, starting with $\ell=0$, we have by induction that

$$
\operatorname{row}_{i}(B) \cap[k+r-1]=\left\{i-r \ell, i+(r-1)+r \ell \mid \ell \in \mathbb{Z}_{\geq 0}\right\} \cap[k+r-1] .
$$

Observe that the first $n-r+1=r(s-1)$ rows of $C_{n}^{r}$ have the cascading structure. We show that if the same number of rows in $A$ have the cascading structure, then $A$ must actually be isomorphic to $C_{n}^{r}$.

Lemma 3.4. If $\operatorname{row}_{i}(A)=[i, i+r)$ for $i \in[r(s-1)]$, then $A \simeq C_{n}^{r}$.

Proof. Permute $A$ and $B$ to have the form described in Lemma 3.3. Since $A$ has dimension $n=r s-1$ and is $r$-regular, the size of $A$ forces $\operatorname{col}_{1}(A)=\{1\} \cup[r(s-1)+1, r s)$ and $\operatorname{col}_{n}(A)=[r(s-1), r s)$. Then by $(1)$,

$$
\left|\operatorname{col}_{i}(B) \cap[r(s-1)+1, r s)\right| \leq 1
$$

for all $i$. For $i \in[r-1]$, Lemma 3.3 implies

$$
\{i, r+i, \ldots, r(s-2)+i\}=\operatorname{col}_{i}(B) \cap[r(s-1)] \text {. }
$$

Since $B$ is $s$-regular, each $\operatorname{col}_{i}(B)$ contains exactly one additional element. By permuting the last $r-1$ rows of $A$ and $B$, we can assume $r(s-1)+i \in \operatorname{col}_{i}(B)$. Taking $1 \leq i<j<r$, observe that $i \in \operatorname{col}_{i}(A) \cap \operatorname{col}_{j}(A) \cap \operatorname{col}_{i}(B)$. This implies $r(s-1)+i \notin \operatorname{col}_{j}(A)$ by the column analog of (1). Now using $r$-regularity of $A$, we must have

$$
[r(s-1)+i, n] \subset \operatorname{col}_{i}(A) \Longrightarrow[i] \subset \operatorname{row}_{r(s-1)+i}(A)
$$

for $i \in[r-1]$. Starting with $i=1$, only columns $[r(s-1)+1, r s-i]$ and rows $[r(s-1)+$ $1, r s-i]$ of $A$ do not have $r$ ones already allocated. By $r$-regularity, we must have

$$
\operatorname{col}_{r s-i}(A)=(r(s-1)-i, r s-i] \quad \text { and } \quad \operatorname{row}_{r s-i}(A)=[r-i] \cup[r s-i, r s) .
$$

This fills row $r s-i$ and column $r s-i$ of $A$. Proceeding inductively for $i=1, \ldots, r-1$, we fill the matrix $A$ and conclude that $A \simeq C_{r s-1}^{r}=C_{n}^{r}$.

The next lemma demonstrates that if $A$ contains the first $n^{\prime}-r+2$ rows of $C_{n^{\prime}}^{r}$ for some $n^{\prime}$, then $n=n^{\prime}$ and $A \simeq C_{n}^{r}$.

Lemma 3.5. If $\operatorname{row}_{i}(A)=[i, i+r)$ for $i \in[k]$ and $\operatorname{row}_{k+1}(A)=\{1\} \cup[k+1, k+r)$, then $k=r(s-1)$ and $A \simeq C_{k+r-1}^{r}$.

Proof. Write $k=r(t-1)+\ell$ for $t \geq 1$ and $0 \leq \ell<r$. Since

$$
[i+1, i+r) \subset \operatorname{row}_{i}(A) \cap \operatorname{row}_{i+1}(A)
$$


for $i \in[k]$, we have by $\operatorname{Remark} 2.2$ that $i \in \operatorname{row}_{i}(B)$ for $i \in[k]$, and $1 \in \operatorname{row}_{k+1}(B)$. By the cascading structure of the first $k$ rows of $A$, we deduce that

$$
\{1, r+1, \ldots, r(t-1)+1, k+1\} \subseteq \operatorname{col}_{1}(B) .
$$

Suppose $\ell>0$. Then $r(t-1)+1$ and $k+1$ are distinct. This contradicts $\mid \operatorname{col}_{k+1}(A) \cap$ $\operatorname{col}_{1}(B) \mid=1$ since $[r(t-1)+1, k+1] \subset \operatorname{col}_{k+1}(A)$. Therefore, $\ell=0$ and $k=r(t-1)$.

We claim that $B$ is $t$-regular. Suppose that $1 \in \operatorname{row}_{i}(B)$ for $i>k+1$. By the cascading structure of the first $k$ rows of $A$, this implies $\{1, r+1, \ldots, r(t-1)+1\} \subseteq \operatorname{row}_{i}(B)$. This contradicts $\left|\operatorname{row}_{k+1}(A) \cap \operatorname{row}_{i}(B)\right|=1$ since $\{1, r(t-1)+1\} \subset \operatorname{row}_{k+1}(A)$. Therefore, $B$ is $t$-regular, $s=t$, and $n=r s-1=k+r-1$.

Lemma 3.4 implies that $A \simeq C_{n}^{r}=C_{k+r-1}^{r}$.

We now use the previous lemmas to present the proof of Lemma 3.2.

Proof of Lemma 3.2. We prove the lemma by induction on the rows of $A$. We can assume $\operatorname{row}_{1}(A)=[r]$ and $\operatorname{row}_{2}(A)=[2, r+1]$. Now suppose $\operatorname{row}_{i}(A)=[i, i+r)$ for all $i \in[\ell]$ and $\ell>1$. Then we apply Lemma 3.3 to assume

$$
\operatorname{row}_{i}(B) \cap[\ell+r-1]=\left\{i-r \mathbb{Z}_{\geq 0}, i+r-1+r \mathbb{Z}_{\geq 0}\right\} \cap[\ell+r-1] .
$$

By assumption, rows $\ell, \ell+1$ are adjacent in $A$ but rows $\ell-1, \ell+1$ are not, so

$$
[\ell, \ell+r-1) \not \subset \operatorname{row}_{\ell-1}(A) \cap \operatorname{row}_{\ell}(A) \cap \operatorname{row}_{\ell+1}(A) .
$$

Therefore, $\ell+r-1 \in \operatorname{row}_{\ell+1}(A)$. Since $\{\ell, \ell+r-1\} \subset \operatorname{row}_{\ell}(B), \ell \notin \operatorname{row}_{\ell+1}(A)$. By adjacency, we deduce that

$$
[\ell+1, \ell+r) \subset \operatorname{row}_{\ell+1}(A) .
$$

Suppose $\operatorname{row}_{\ell+1}(A)=\{i\} \cup[\ell+1, \ell+r)$ for $i<\ell$. Then rows $[i, \ell+1]$ satisfy Lemma 3.5. Therefore, we either get a contradiction or $A \simeq C_{n}^{r}$.

Otherwise $\operatorname{row}_{\ell+1}(A) \not \subset[\ell+r-1]$, and we can permute columns to assume $\operatorname{row}_{\ell+1}(A)=$ $[\ell+1, \ell+r]$. This completes the inductive step. Hence either $A \simeq C_{n}^{r}$ or we can permute the columns of $A$ such that $\operatorname{row}_{i}(A)=[i, i+r)$ for $i \in[k]$.

Corollary 3.6. Suppose $G_{A}$ contains a cycle where vertices of distance 2 apart in the cycle are not adjacent in $G_{A}$. Then $A \simeq C_{n}^{r}$.

Proof. Let rows $[k]$ correspond to the vertices of the cycle. We must have $k>3$ in order for the assumptions to hold. Suppose $A \not C_{n}^{r}$. Then by Lemma 3.2, $\operatorname{row}_{i}(A)=[i, i+r)$ for $i \in[k]$. Since $\left|\operatorname{row}_{1}(A) \cap \operatorname{row}_{k}(A)\right|=\max (r-k+1,0)<r-2$, rows 1 and $k$ cannot be adjacent, which is a contradiction. 


\subsection{Cliques}

In the previous section we considered triangle-free paths in the graph $G_{A}$. We now look at the structure of triangles, and in greater generality, cliques in $G_{A}$. In particular, we provide a lemma analogous to Lemma 3.2 for cliques.

Lemma 3.7. If rows $[k]$ form a $k$-clique in $G_{A}$, then the columns of $A$ can be permuted such that $\operatorname{row}_{i}(A)=[r-1] \cup\{r+i-1\}$ and $\{i, r+i-1\} \subset \operatorname{row}_{i}(B)$ for $i \in[k]$.

Proof. Permute the columns so $\operatorname{row}_{1}(A)=[r]$ and $\{1, r\} \subset \operatorname{row}_{1}(B)$. Thus for $i>1$,

$$
\left|\operatorname{row}_{i}(A) \cap\{1, r\}\right| \leq 1 \text {. }
$$

Since each row $i \in(1, k]$ is adjacent to row 1 , we have either

$$
[r-1] \subset \operatorname{row}_{i}(A) \quad \text { or } \quad[2, r] \subset \operatorname{row}_{i}(A) .
$$

By possibly switching columns 1 and $r$, we may assume without loss of generality that $[r-1] \subset \operatorname{row}_{2}(A)$. Suppose $[2, r] \subset \operatorname{row}_{i}(A)$ for some $i>2$. Since $\{1,2, i\} \subset \operatorname{col}_{2}(A)$ and $1 \in \operatorname{col}_{r}(B)$, we deduce that $r \notin \operatorname{row}_{i}(B)$. Since rows 2 and $i$ must be adjacent,

$$
\operatorname{row}_{i}(A) \backslash\{r\} \subset \operatorname{row}_{2}(A) .
$$

Thus, $\operatorname{row}_{i}(B)$ must contain two elements in $\operatorname{row}_{2}(A)$, which is a contradiction. Therefore, $[r-1] \subset \operatorname{row}_{i}(A)$ for $i \in[k]$. No two rows of $A$ may be equal, so we can permute columns $[r, n]$ to assume

$$
\operatorname{row}_{i}(A)=[r-1] \cup\{r+i-1\} .
$$

Since $[r-1] \subset \operatorname{row}_{1}(A) \cap \operatorname{row}_{i}(A)$, we must have $r+i-1 \in \operatorname{row}_{i}(B)$ by (1). Additionally, we know that

$$
[k] \subset \bigcap_{i=1}^{r-1} \operatorname{col}_{i}(A),
$$

so no column of $B$ can have two 1s in the first $k$ rows. We may therefore permute the first $r-1$ columns of $A$ and $B$ simultaneously to assume $\{i, r+i-1\} \subset \operatorname{row}_{i}(B)$.

Example. We give an example of the rows in $A$ and $B$ corresponding to a clique in $G_{A}$. Here $r=4$ and $k=3$. The diagram on the left shows $\operatorname{row}_{i}(A)$ and the diagram on the right shows $\operatorname{row}_{i}(B) \cap[k+r-1]$ for $i \in[k]$.
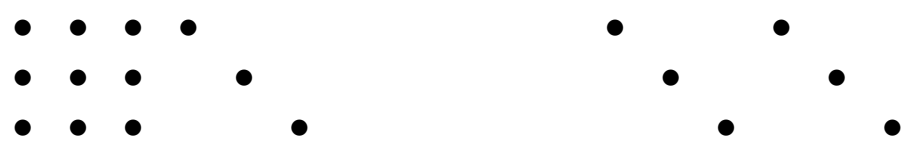

Remark 3.8. Suppose rows $[k]$ form a clique in $G_{A}$. By Lemma 3.7, we can permute columns to get $\operatorname{row}_{i}(A)=[r-1] \cup\{r+i-1\}$ for $i \in[k]$. Then

$$
[r-1]=\operatorname{row}_{1}(A) \cap \operatorname{row}_{2}(A)=\bigcap_{i=1}^{k} \operatorname{row}_{i}(A) .
$$




\subsection{Connected components}

We define a clique tree as follows. Start with a tree $T$. Create a new graph equal to the disjoint union of $|V(T)|$ cliques of arbitrary size. For each edge $i j \in E(T)$, choose one vertex in clique $i$ and one vertex in clique $j$ of the new graph, and combine the two chosen vertices into one vertex. We additionally require that the new graph does not contain a vertex incident to more than two maximal cliques. We call the resulting graph a clique tree. Note that a triangle-free clique tree is a path.

In this section, we show that if $A \not C_{n}^{r}$, then the connected components of $G_{A}$ must be clique trees. Moreover, connected components containing a triangle must contain fewer than $r$ vertices. At the end of the section we prove that a connected component of $G_{A}$ uniquely determines, up to permutation of the columns, the corresponding rows of $A$.

Lemma 3.9. Suppose rows $1,3,4$ are all adjacent to row 2 in $A$. Then two rows in $\{1,3,4\}$ must be adjacent.

Proof. Suppose rows 1 and 3 are not adjacent. Then using Lemmas 3.2 and 3.3, we can permute columns such that $\operatorname{row}_{i}(A)=[i, i+r)$ for $i \in[3]$ and $\{2, r+1\} \subset \operatorname{row}_{2}(B)$. Since rows 2 and 4 are adjacent in $A$, we must have either $[2, r] \subset \operatorname{row}_{4}(A)$ or $[3, r+1] \subset \operatorname{row}_{4}(A)$. Therefore, row 4 is adjacent to row 1 or 3 in $A$.

Note that the lemma implies that the only possible trees in $G_{A}$ are paths. We next prove that if a vertex is adjacent to two vertices of a clique in $G_{A}$, then it must be adjacent to every vertex in the clique.

Lemma 3.10. Suppose rows $[k]$ of $A$ form a clique in $G_{A}$, and row $k+1$ is adjacent to rows 1 and 2 in $G_{A}$. Then row $k+1$ is adjacent to every row $i$ for $i \in[k]$.

Proof. Lemma 3.7 implies that $\operatorname{row}_{i}(A)=[r-1] \cup\{r+i-1\}$ for $i \in[k]$. Observe that since rows $1,2, k+1$ form a triangle, (6) implies that

$$
[r-1]=\operatorname{row}_{1}(A) \cap \operatorname{row}_{2}(A) \subset \operatorname{row}_{k+1}(A) .
$$

Therefore, row $k+1$ is adjacent to every row $i$ for $i \in[k]$.

Observe that if two cliques share at least two vertices, Lemma 3.10 shows that their union must also be a clique. Now Lemma 3.9 implies that any vertex is incident to at most two maximal cliques. The previous two lemmas show that $G_{A}$ essentially contains only paths and cliques. The next proposition will show that a connected component of $G_{A}$ for $A \not C_{n}^{r}$ must indeed be a clique tree.

Lemma 3.11. If $G_{A}$ has a cycle that is not contained inside a clique, then $A \simeq C_{n}^{r}$.

Proof. Suppose $G_{A}$ contains such a cycle, and let the vertices of the cycle be $[k]$. If $k>3$ and there exists a row $i \in[k]$ in the cycle with cyclically shifted rows $i-1$ and $i+1$ adjacent in $G_{A}$, consider instead the cycle with vertices $[k] \backslash\{i\}$. Repeating, we either reduce the cycle to a triangle or a cycle where vertices of distance 2 apart are not 
adjacent. If the reduced cycle is a triangle, Lemma 3.10 implies that the original rows $[k]$ form a clique. Otherwise, the reduced cycle satisfies the conditions of Corollary 3.6, so $A \simeq C_{n}^{r}$.

Combining Lemmas 3.9, 3.10, and 3.11, we conclude the following theorem.

Theorem 3.12. If $A \not C_{n}^{r}$, then each connected component of $G_{A}$ is a clique tree.

Corollary 3.13. If $G_{A}$ is triangle-free, then either $A \simeq C_{n}^{r}$ or $G_{A}$ is a disjoint union of paths.

We next give a bound on the size of connected components that do contain triangles.

Lemma 3.14. If a connected component of $G_{A}$ contains a triangle, then the component has fewer than $r$ vertices.

Proof. Suppose a connected component contains at least $r$ vertices. We can then choose a subset of $r$ vertices $W \subset V\left(G_{A}\right)$ such that the subgraph of $G_{A}$ induced by $W$ is connected and contains a triangle. Rearrange the rows so that $W=[r]$, and each row $j \in W \backslash\{1\}$ is adjacent to some $i<j$. Let $t_{i} \in W$ for $i \in[3]$ induce a triangle, with $t_{1}<t_{2}<t_{3}$.

We claim that

$$
\left|\bigcap_{i=1}^{k} \operatorname{row}_{i}(A)\right| \geq r-(k-1)
$$

for $k \in[r]$. We prove this by induction. The case $k=1$ is clear. Now assume the claim is true for some $k$. Since $\left|\operatorname{row}_{k+1}(A) \cap \operatorname{row}_{i}(A)\right|=r-1$ for some $i \leq k$, there is at most one element of $\operatorname{row}_{i}(A)$ that is not in $\operatorname{row}_{k+1}(A)$. Consequently there is at most one element of $\bigcap_{i=1}^{k} \operatorname{row}_{i}(A)$ that is not in $\operatorname{row}_{k+1}(A)$. Thus,

$$
\left|\bigcap_{i=1}^{k+1} \operatorname{row}_{i}(A)\right| \geq r-(k-1)-1=r-((k+1)-1),
$$

proving the claim.

Therefore, $\left|\bigcap_{i=1}^{r} \operatorname{row}_{i}(A)\right| \geq 1$, with equality if and only if

$$
\bigcap_{i=1}^{k} \operatorname{row}_{i}(A) \neq \bigcap_{i=1}^{k+1} \operatorname{row}_{i}(A)
$$

for every $k \in[r-1]$. Since $t_{1}, t_{2}, t_{3}$ induce a triangle in $G_{A},(6)$ implies that $\operatorname{row}_{t_{1}}(A) \cap$ $\operatorname{row}_{t_{2}}(A) \subset \operatorname{row}_{t_{3}}(A)$. Thus, (7) does not hold for $k=t_{3}-1$. Therefore,

$$
\left|\bigcap_{i=1}^{r} \operatorname{row}_{i}(A)\right|>1
$$

Thus, there exist two columns of $A$ with $r$ ones in the same rows. This is a contradiction since $A$ is invertible. 
Corollary 3.15. The graph $G_{A}$ is connected if and only if $A \simeq C_{n}^{r}$.

Proof. Suppose $G_{A}$ is connected and not isomorphic to $C_{n}^{r}$. Since $n=r s-1>r$, Lemma 3.14 implies $G_{A}$ is triangle-free. By Corollary $3.13, G_{A}$ is a disjoint union of paths. If $G_{A}$ is a single path, Lemma 3.2 allows us to permute $A$ such that $\operatorname{row}_{i}(A)=[i, i+r)$ for $i \in[n]$. Since $A$ is a square matrix, this is impossible.

The other direction is obvious.

We now present the proof of our main result relating induced Johnson subgraphs and Lehman matrices.

Proof of Theorem 3.1. We may assume that $W=[k]$. If $W$ induces a path, then Lemma 3.2 proves the claim. Otherwise, the connected component contains a triangle, so Lemma 3.14 implies $k<r$. From the proof of Lemma 3.14, we know that $\left|\bigcap_{i=1}^{k} \operatorname{row}_{i}(A)\right| \geq 2$. Therefore, there exist two columns of $A$ that have $1 \mathrm{~s}$ in rows $[k]$. Then (1) implies that

$$
\left|\operatorname{col}_{i}(B) \cap[k]\right| \leq 1
$$

for all $i$. Rearrange $W$ so that each row $j \in W \backslash\{1\}$ is adjacent to some $i<j$. We will prove inductively that for $\ell \in[k]$, the columns of $A$ can be permuted such that

1. $\operatorname{row}_{\ell}(A) \subset[\ell+r-1]$,

2. $[\ell, r) \subset \bigcap_{i=1}^{\ell} \operatorname{row}_{i}(A)$,

3. $\{\ell, \ell+r-1\}=\operatorname{row}_{\ell}(A) \cap \operatorname{row}_{\ell}(B)$, and

4. rows $[\ell]$ are unique up to permutations of columns.

The claim is trivial for $\ell \leq 2$. Now assume the claim is true for all $i \leq \ell$ for some $\ell \in[2, k)$. By statement 3 of the inductive hypothesis,

$$
i \in \operatorname{col}_{i}(B) \cap \operatorname{col}_{i+r-1}(B)
$$

for $i \in[\ell]$. Therefore, (8) implies that $\ell+1 \notin \operatorname{col}_{i}(B)$ for $i \in[\ell] \cup[r, r+\ell)$, or equivalently $\operatorname{row}_{\ell+1}(B)$ is disjoint from $[\ell] \cup[r, r+\ell)$. Since $[\ell, r) \subset \bigcap_{i=1}^{\ell} \operatorname{row}_{i}(A),(1)$ implies that

$$
\left|\operatorname{row}_{\ell+1}(B) \cap[\ell, r)\right| \leq 1 .
$$

Thus, $\operatorname{row}_{\ell+1}(A) \cap \operatorname{row}_{\ell+1}(B) \not \subset[r+\ell-1]$ because the intersection has size 2 .

There exists $i \in[\ell]$ such that rows $i$ and $\ell+1$ in $A$ are adjacent. Thus,

$$
\left|\operatorname{row}_{\ell+1}(A) \backslash \operatorname{row}_{i}(A)\right|=1,
$$

so row $\ell+1$ in $A$ has exactly one 1 outside $[r+\ell-1]$. We can permute columns to assume

$$
r+\ell \in \operatorname{row}_{\ell+1}(A) \cap \operatorname{row}_{\ell+1}(B) .
$$


Thus, $\operatorname{row}_{\ell+1}(A) \subset[r+\ell]$, proving statement 1 . Since rows $i$ and $\ell+1$ are adjacent in $A$ and $\{i, i+r-1\} \subset \operatorname{row}_{i}(A) \cap \operatorname{row}_{i}(B)$, we have either

$$
\operatorname{row}_{\ell+1}(A)=\operatorname{row}_{i}(A) \cup\{r+\ell\} \backslash\{i\} \text { or } \operatorname{row}_{\ell+1}(A)=\operatorname{row}_{i}(A) \cup\{r+\ell\} \backslash\{i+r-1\} .
$$

Note that since $[\ell, r) \subset \operatorname{row}_{i}(A)$, in both cases $[\ell+1, r) \subset \operatorname{row}_{\ell+1}(A)$, asserting statement 2. We showed above that $\operatorname{row}_{\ell+1}(B)$ is disjoint from $[\ell] \cup[r, r+\ell)$. Since $r+\ell \in \operatorname{row}_{\ell+1}(B)$ and $\operatorname{row}_{\ell+1}(A) \subset[r+\ell]$, we deduce that

$$
\left|\operatorname{row}_{\ell+1}(B) \cap[\ell+1, r)\right|=1 .
$$

Since $[\ell+1, r) \subset \bigcap_{i=1}^{\ell+1} \operatorname{row}_{i}(A)$, we can permute the columns $[\ell+1, r)$ of $A$ and $B$ simultaneously to assume $\ell+1 \in \operatorname{row}_{\ell+1}(B)$, leaving the first $\ell+1$ rows of $A$ unchanged. We now have $\{\ell+1, \ell+r\} \subset \operatorname{row}_{\ell+1}(A) \cap \operatorname{row}_{\ell+1}(B)$, proving statement 3 .

To show uniqueness, we only need to show exactly one case in (9) is true. Take some row $j$ such that rows $i$ and $j$ are adjacent in $G_{A}$ and $j \leq \ell$ (this is possible since $\ell \geq 2$ and rows 1 and 2 are adjacent). Let

$$
\{a\}=\{i, i+r-1\} \cap \operatorname{row}_{j}(A)=\operatorname{row}_{i}(A) \cap \operatorname{row}_{i}(B) \cap \operatorname{row}_{j}(A),
$$

which is a one element set since $\left|\operatorname{row}_{i}(A) \cap \operatorname{row}_{j}(A)\right|=r-1$ implies $\mid\{i, i+r-1\} \cap$ $\operatorname{row}_{j}(A) \mid \geq 1$ and $\left|\operatorname{row}_{i}(B) \cap \operatorname{row}_{j}(A)\right|=1$ implies $\left|\{i, i+r-1\} \cap \operatorname{row}_{j}(A)\right| \leq 1$. Let

$$
\{b\}=\{i, i+r-1\} \backslash\{a\} .
$$

Since rows $i, j$ are adjacent, we have $\operatorname{row}_{i}(A) \cap \operatorname{row}_{j}(A)=\operatorname{row}_{i}(A) \backslash\{b\}$. If $\operatorname{row}_{\ell+1}(A)=$ $\operatorname{row}_{i}(A) \cup\{r+\ell\} \backslash\{b\}$, then rows $j, \ell+1$ are also adjacent. If rows $j, \ell+1$ are adjacent in $G_{A}$, then rows $i, j, \ell+1$ form a triangle, so

$$
a \in \operatorname{row}_{i}(A) \cap \operatorname{row}_{j}(A) \subset \operatorname{row}_{\ell+1}(A)
$$

by (6). Therefore,

$$
\operatorname{row}_{\ell+1}(A)=\operatorname{row}_{i}(A) \cup\{r+\ell\} \backslash\{b\}
$$

if and only if rows $j, \ell+1$ are adjacent in $G_{A}$.

Thus, exactly one case in (9) can be true, and we conclude that the rows $[\ell+1]$ are uniquely determined by the graph $G_{A}$ up to permutations of the columns. This completes the inductive step and proves the theorem.

\section{Bounds on clique size and degree}

In this section, we continue to assume that $A$ is an $r$-regular thin Lehman matrix of dimension $n \times n, B$ is its $s$-regular dual, and $n=r s-1$. In Section 3, we showed that either $A \simeq C_{n}^{r}$ or the connected components of $G_{A}$ are clique trees. We are therefore interested in the maximum size of cliques in $G_{A}$, which would give us a better idea of the possible structures of the induced Johnson subgraph.

We provide a sharp upper bound on the maximum clique size of the graph $G_{A}$, and give a relation between the clique sizes of $G_{A}$ and $G_{B^{T}}$. We also give an upper bound on the maximum degree of $G_{A}$. 


\subsection{Maximum clique size}

Let the clique number $\omega\left(G_{A}\right)$ denote the number of vertices in a maximal clique in $G_{A}$. The equation $A B^{T}=J+I$ gives a condition on the size of cliques in $G_{A}$ and $G_{B^{T}}$.

Lemma 4.1. The clique numbers $\omega\left(G_{A}\right)$ and $\omega\left(G_{B^{T}}\right)$ are equal.

Proof. Let $\omega\left(G_{A}\right)=k$. Use Lemma 3.7 to permute rows/columns such that

$$
\operatorname{row}_{i}(A)=[r-1] \cup\{i+r-1\} \quad \text { and } \quad\{i, i+r-1\} \subset \operatorname{row}_{i}(B)
$$

for $i \in[k]$. Now consider any row $i>k$ with $r \in \operatorname{row}_{i}(B)$. Then $\left|\operatorname{row}_{1}(A) \cap \operatorname{row}_{i}(B)\right|=1$ implies $[r-1] \cap \operatorname{row}_{i}(B)=\emptyset$. Now for $j \in(1, k],\left|\operatorname{row}_{j}(A) \cap \operatorname{row}_{i}(B)\right|=1 \operatorname{implies}$ $j+r-1 \in \operatorname{row}_{i}(B)$. Thus, $[r, r+k) \subset \operatorname{row}_{i}(B)$. Since $[k] \subset \operatorname{col}_{i}(A)$ for $i \in[r-1],(1)$ implies that $\left|\operatorname{col}_{r}(B) \cap[k]\right|=1$. By $s$-regularity of $B$, there must be $s-1$ rows $i>k$ with $r \in \operatorname{row}_{i}(B)$. Consequently there are $s-1$ rows of $B$ containing columns $[r, r+k)$. These columns form a $k$-clique in $G_{B^{T}}$.

This shows that $\omega\left(G_{A}\right) \leq \omega\left(G_{B^{T}}\right)$. Since $A B^{T}=B^{T} A=J+I$ by Theorem 2.1, we get equality via symmetry.

Corollary 4.2. The maximum clique size $\omega\left(G_{A}\right) \leq \min (r-1, s-1)$.

Proof. Lemma 3.14 implies $\omega\left(G_{A}\right) \leq r-1$ and $\omega\left(G_{B^{T}}\right) \leq s-1$. It follows that

$$
\omega\left(G_{A}\right)=\omega\left(G_{B^{T}}\right) \leq \min (r-1, s-1) .
$$

We will show that the previous upper bound is sharp. For $r=s$ and $n=r^{2}-1$, define the block matrix

$$
\Omega_{r}=\left[\begin{array}{cccccc}
J_{r-1} & E_{11} & E_{22} & \ldots & E_{r-1, r-1} & 0 \\
0 & J_{r-1} & E_{11} & & E_{r-2, r-2} & E_{r-1, r-1} \\
E_{r-1, r-1} & 0 & J_{r-1} & & E_{r-3, r-3} & E_{r-2, r-2} \\
\vdots & & & \ddots & & \vdots \\
E_{22} & E_{33} & E_{44} & & J_{r-1} & E_{11} \\
E_{11} & E_{22} & E_{33} & \ldots & 0 & J_{r-1}
\end{array}\right]
$$

where each block is $(r-1) \times(r-1), J_{r-1}$ is the matrix of all $1 \mathrm{~s}$, and $E_{i j}$ is the matrix with a single 1 in row $i$, column $j$. Observe that $G_{\Omega_{r}}=\bigsqcup^{r+1} K_{r-1}$, where $K_{r-1}$ is the complete graph with $r-1$ vertices. For this reason, we will call $\Omega_{r}$ a clique matrix. In [8], it is shown that $\Omega_{r}$ is a thin Lehman matrix for $r \geq 3$. For completeness, we give a sketch of the proof.

Lemma 4.3 ([8], Lemma 4). For $r \geq 3$, the clique matrix $\Omega_{r}$ is a thin Lehman matrix.

Proof. Let $\pi \in S_{r+1}$ be the cycle permutation sending

$$
\pi: 1 \mapsto 2, \ldots, r \mapsto r+1, r+1 \mapsto 1
$$


For $(i, j) \in[r+1] \times[r-1]$, define the 0,1 vector $y^{i j}$ to be the incidence vector of

$$
\left\{\left(\pi^{k}(i)-1\right)(r-1)+k,\left(\pi^{r}(i)-1\right)(r-1)+j \mid k=1, \ldots, r-1\right\} .
$$

By letting $B$ be the $n \times n$ matrix with row $(i-1)(r-1)+j$ equal to $y^{\pi^{j+1}(i), j}$, it can be checked that $\Omega_{r} \cdot B^{T}=J+I$.

Next, we consider the case when $r<s$.

Proposition 4.4. For any $3 \leq r<s$, there exists an $r$-regular Lehman matrix $A$ such that $\omega\left(G_{A}\right)=r-1$, i.e., A contains $J_{r-1}$ as a submatrix.

Proof. Define the $n \times n$ matrix $A_{0}$ to be

$$
A_{0}=\left[\begin{array}{c|cccccc|c}
J_{r-1} & E_{11} & E_{21} & E_{31} & \ldots & E_{r-2,1} & E_{r-1,1} & \\
\hline E_{11} & J_{r-1} & E_{22} & E_{33} & \ldots & E_{r-2, r-2} & 0 \\
E_{12} & 0 & J_{r-1} & E_{22} & & E_{r-3, r-3} & E_{r-2, r-2} \\
E_{13} & E_{r-2, r-2} & 0 & & & & \vdots \\
\vdots & \vdots & & & & & E_{22} \\
E_{1, r-1} & E_{22} & \ldots & & E_{r-2, r-2} & 0 & J_{r-1} & \\
\hline & & & & & & & C
\end{array}\right],
$$

where $J_{r-1}$ is the $(r-1) \times(r-1)$ matrix of all $1 \mathrm{~s}, E_{i j}$ is the $(r-1) \times(r-1)$ matrix with a single 1 in row $i$, column $j$, and $C$ is the circulant matrix $C_{(s-r+1) r}^{r}$ with the first column and last row removed. The lines are placed to emphasize the matrix pattern.

Let $B_{1}(i, j):=(i-1)(r-1)+j$ and $B_{2}(i, j):=r(r-1)+r(i-1)+j$. Define the $n \times n$ matrix $\Sigma$ with 1s only at

$$
\begin{aligned}
& \left.\left(B_{1}(2, r-1), B_{2}(s-r+1, r-1)\right),\left(B_{1}(r+1-i, r-1)\right), B_{2}(1, i)\right), \\
& \left(B_{2}(1,1), B_{1}(r, r-1)\right),\left(B_{2}(s-r+1, r-i), B_{1}(i+1, r-1)\right)
\end{aligned}
$$

for $i \in[r-2]$. We claim that $A:=A_{0}+\Sigma$ is an $r$-regular thin Lehman matrix.

We provide the rows of $B$. For convenience, given a subset $S \subset[n]$, let $\operatorname{vec}(S)$ denote the incidence vector in $\{0,1\}^{n}$ of $S$. Let $e_{i}:=\operatorname{vec}\{i\}$. Define

$$
x_{i}=\operatorname{vec}\left\{B_{1}(2,1), \ldots, B_{1}(r, 1), B_{2}(1, i), \ldots, B_{2}(s-r+1, i)\right\}
$$

for $i \in[r-1]$. Let $\tau \in S_{r-1}$ be the cyclic permutation sending $1 \mapsto 2, \ldots, r-1 \mapsto 1$. For $(i, j) \in[r-1] \times[r-1]$, we define

$$
\begin{aligned}
y_{i j}=\operatorname{vec}\left\{B_{1}(1, i), B_{1}(1+\tau(i), 2), \ldots, B_{1}\left(1+\tau^{r-3}(i), r-2\right),\right. \\
\\
\left.B_{1}\left(1+\tau^{-1}(i), j\right), B_{2}(1, r-i), \ldots, B_{2}(s-r+1, r-i)\right\} .
\end{aligned}
$$

Lastly for $i \in[r-1]$, define

$$
z_{i}=\operatorname{vec}\left\{B_{1}(1, i), B_{1}(2, r-1), \ldots, B_{1}(r, r-1), B_{2}(1, r), \ldots, B_{2}(s-r, r)\right\} .
$$


Now let the rows of $B$ equal

$$
\begin{array}{ll}
x_{i} & \text { for } i \in[r-1], \\
x_{r-1}+\sum_{j=k}^{s-r+1}\left(e_{B_{2}(j, r-2)}-e_{B_{2}(j, r-1)}\right) & \text { for } k \in[2, s-r+1], \\
y_{i j} & \text { for }(i, j) \in[r-1] \times[r-1], \\
y_{1, r-1}+\sum_{j=1}^{k}\left(e_{B_{2}(j, r)}-e_{B_{2}(j, r-1)}\right) & \text { for } k \in[s-r], \\
y_{i, r-1}+\sum_{j=k}^{s-r+1}\left(e_{B_{2}(j, r-i-1)}-e_{B_{2}(j, r-i)}\right) & \text { for }(i, k) \in[2, r-1] \times[2, s-r+1], \text { and } \\
z_{i} & \text { for } i \in[r-1] .
\end{array}
$$

There are

$$
(r-1)+(s-r)+(r-1)(r-1)+(s-r)+(r-2)(s-r)+(r-1)=r s-1=n
$$

rows in total, so $B$ is an $n \times n$ matrix. To avoid excessive technical details, we leave it to the reader to check that $A B^{T}=J+P$ for some permutation matrix $P$. Thus, $A(P B)^{T}=J+P P^{T}=J+I$. We conclude that $A$ is a thin Lehman matrix.

Example. We provide an example of the matrices $A$ and $B$ described in Proposition 4.4 such that $A B^{T}=J+I$. In the example $r=4, s=5$, and $n=19$.
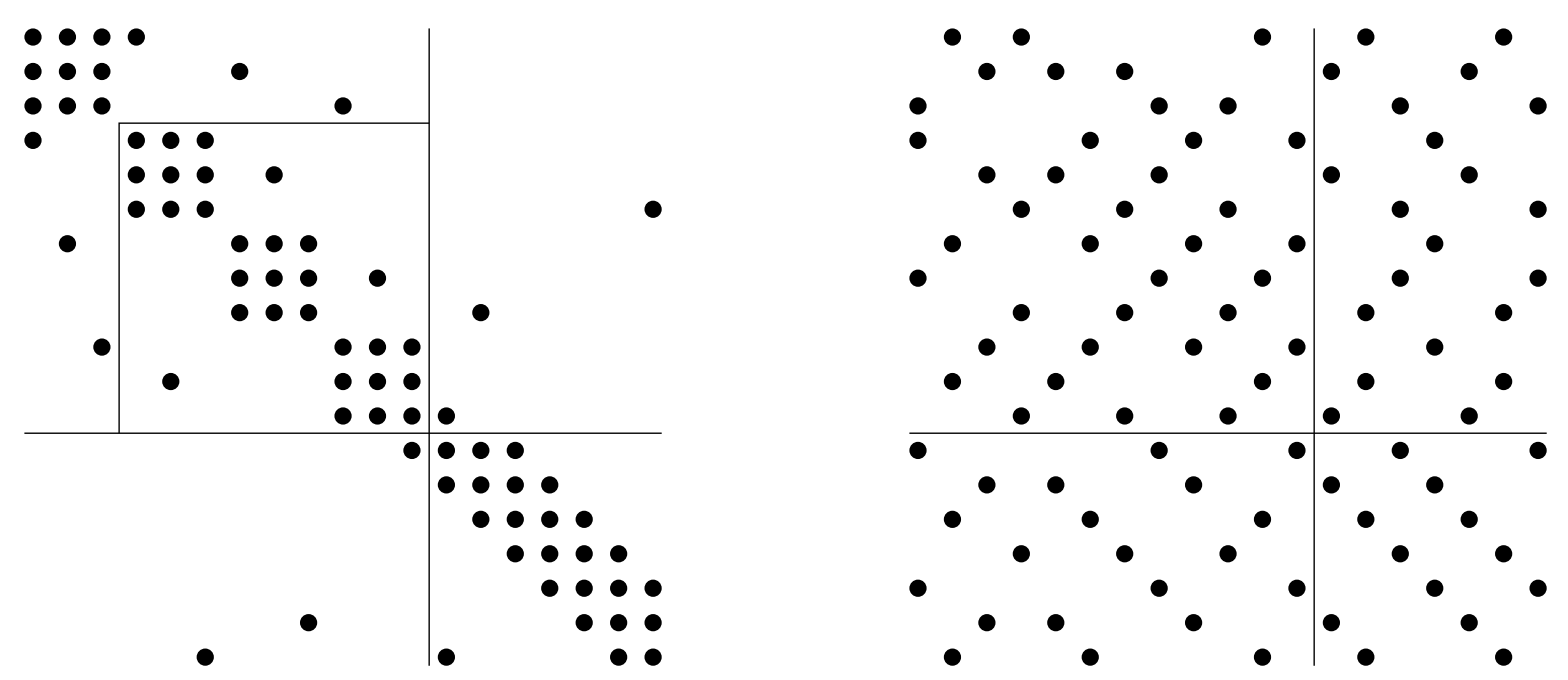

Proposition 4.4 shows that Corollary 4.2 is sharp for $r<s$. Since $\omega\left(G_{A}\right)=\omega\left(G_{B}^{T}\right)$ by Lemma 4.1, taking $B^{T}$ from Proposition 4.4 shows the bound is sharp for $r>s$. The clique matrix $\Omega_{r}$ shows sharpness for $r=s$, so we conclude that $\omega\left(G_{A}\right) \leq \min (r-1, s-1)$ is sharp in general. 


\subsection{Maximum degree}

We provide a relation between the maximum degree and the maximum clique size of the induced Johnson subgraph. Using bounds on maximum clique size, we give a bound on the maximum degree of $G_{A}$. We do not, however, believe this bound is sharp.

Lemma 4.5. The maximum degree $\Delta\left(G_{A}\right) \leq 2\left(\omega\left(G_{A}\right)-1\right)$.

Proof. If $A \simeq C_{n}^{r}$, then $\Delta\left(G_{A}\right)=2$ and $\omega\left(G_{A}\right)=2$, so the lemma holds. If $A \not C_{n}^{r}$, Theorem 3.12 says that each connected component of $G_{A}$ is a clique tree. Take a vertex $i$ of $G_{A}$. By the definition of a clique tree, $i$ is incident to at most two maximal cliques. The degree of any vertex in a maximal clique of $G_{A}$ is $\omega\left(G_{A}\right)-1$. Therefore, the degree of $i$ is at most $2\left(\omega\left(G_{A}\right)-1\right)$.

Observe that in the case $r=3$, Corollary 4.2 says $\omega\left(G_{A}\right) \leq 2$, so the previous lemma implies that $\Delta\left(G_{A}\right) \leq 2$.

Proposition 4.6. For $r>3$, the maximum degree $\Delta\left(G_{A}\right) \leq \min (r-2,2(s-2))$.

Proof. Assume $\operatorname{row}_{1}(A)=[r]$, and suppose that the $r-1$ rows $[2, r]$ are all adjacent to row 1 . We may also assume by permuting columns that $\{1,2\} \subset \operatorname{row}_{1}(B)$, which implies that $[3, r] \subset \operatorname{row}_{i}(A)$ for $i \in[r]$ by adjacency. Thus,

$$
\operatorname{col}_{3}(A)=\operatorname{col}_{4}(A)=[r]
$$

which contradicts $A$ being invertible. Therefore, $\Delta\left(G_{A}\right) \leq r-2$. It follows from Corollary 4.2 and Lemma 4.5 that $\Delta\left(G_{A}\right) \leq 2(s-2)$.

\section{Classification of graphs with two connected com- ponents}

In Section 3, we showed that a Lehman matrix $A$ has a connected graph $G_{A}$ if and only if $A \simeq C_{n}^{r}$. We now classify all Lehman matrices such that the graph $G_{A}$ has exactly two connected components. Recall that a Lehman matrix $A$ with $d>1$ has no edges in $G_{A}$. Therefore, we may assume $A$ is an $r$-regular thin Lehman matrix, $B$ is the $s$-regular dual, and $n=r s-1$.

Theorem 5.1. For any $r, s>2$, a graph $G$ with $n$ vertices and two connected components is the Johnson subgraph $G_{A}$ associated to an r-regular Lehman matrix $A$ if and only if each component of $G$ is a path with length greater than $r$ and not equivalent to $-1,0$ $(\bmod r)$. Furthermore the matrix $A$ is determined, up to isomorphism, by the graph $G$.

Proof. ( $\Longrightarrow$ ) Suppose a component of $G_{A}$ has length greater than $n-r>r$. Then it must be either a path or a cycle by Lemma 3.14. Lemmas 3.2 and 3.4 imply that $A \simeq C_{n}^{r}$. Since $G_{A}$ has two connected components, we must have that the number of vertices in each component is at most $n-r$. Equivalently, each component has at least $r$ vertices and must be a path by Lemma 3.14 . 
Claim 1. Neither path has length equivalent to $-1,0(\bmod r)$.

Let the lengths of the two paths be $k$ and $n-k$. Since $k+(n-k)=n \equiv-1(\bmod r)$, we can assume the length of one of the paths is

$$
k=r \ell+j
$$

for $\ell \geq 1$ and $0 \leq j<r / 2$. By Lemmas 3.2 and 3.3, we rearrange rows and columns to get $\operatorname{row}_{i}(A)=[i, i+r)$ and

$$
\operatorname{row}_{i}(B) \cap[k+r-1]=\left\{i-r \mathbb{Z}_{\geq 0}, i+r-1+r \mathbb{Z}_{\geq 0}\right\} \cap[k+r-1]
$$

for $i \in[k]$. Lemma 3.2 also implies that there exists a permutation $\sigma \in S_{n}$ such that

$$
\operatorname{row}_{k+i}(A)=\sigma([i, i+r)) \quad \text { for } \quad i \in[n-k] .
$$

Additionally since $n-k>r$, we may assume

$$
\{\sigma(i), \sigma(i+r-1)\}=\operatorname{row}_{k+i}(B) \cap \sigma([i, i+r))
$$

for $i \in[n-k]$ from the proof of Lemma 3.3.

Observe that

$$
\begin{aligned}
\left|\operatorname{col}_{i}(A) \cap[k]\right| & =\min (i, k+r-i, r) & & \text { for } i \in[k+r-1], \text { and } \\
\left|\operatorname{col}_{\sigma(i)}(A) \cap(k, n]\right| & =\min (i, n-k+r-i, r) & & \text { for } i \in[n-k+r-1] .
\end{aligned}
$$

Since $A$ is $r$-regular, we must have

$$
\left|\operatorname{col}_{\sigma(i)}(A) \cap[k]\right|+\left|\operatorname{col}_{\sigma(i)}(A) \cap(k, n]\right|=\min (\sigma(i), k+r-\sigma(i), r)+\min (i, n-k+r-i, r)=r .
$$

From this observation we deduce that

$$
\sigma(i) \in\{r-i, k+i\}
$$

for $i \in[r-1]$. If $\sigma(1)=r-1$, we can swap rows $i, k-i$ for $i \in[k]$ and columns $i, k+r-i$ for $i \in[k+r-1]$ in $A$ and $B$ simultaneously to assume that $\sigma(1)=k+1$ and $k+1 \in \operatorname{row}_{k+1}(A) \cap \operatorname{row}_{k+1}(B)$. Due to the cascading structure of rows $[k]$ of $A$, we have that

$$
\left\{k+1-r \mathbb{Z}_{\geq 0}\right\} \cap[k] \subset \operatorname{row}_{k+1}(B),
$$

so $j+1 \in \operatorname{row}_{k+1}(B)$. Since $\{j+1, k+1\} \subset \operatorname{col}_{j+1}(B)$, Remark 2.2 says that

$$
\left|\{j+1, k+1\} \cap \operatorname{col}_{i}(A)\right| \leq 1
$$

for columns $i>j+1$. As $\operatorname{row}_{j+1}(A)=[j+1, j+r]$, we deduce that

$$
[j+2, j+r] \cap \operatorname{row}_{k+1}(A)=\emptyset .
$$


Noting that $\operatorname{row}_{k+1}(A)=\sigma([1, r])$, if $\sigma(r-i)=i$ for any $i \in[j+2, r)$, then

$$
\sigma([1, r]) \cap[j+2, r) \neq \emptyset
$$

which gives a contradiction. Therefore, (15) implies that $\sigma(r-i)=k+r-i$ for $i \in[j+2, r)$. Thus,

$$
[k+1, k+r-j-2] \subset \operatorname{row}_{k+1}(A) .
$$

Note that $\sigma(r-j-1) \notin \operatorname{row}_{k+1}(B)$ from (12). Therefore, $\sigma(r-j-1) \neq j+1$, which forces $\sigma(r-j-1)=k+r-j-1$. If $j=0$, we have shown that $[k+1, k+r) \subset \operatorname{row}_{k+1}(A)$, which contradicts rows $k$ and $k+1$ not being adjacent in $G_{A}$. Therefore, $k \neq \equiv 0(\bmod r)$ and since $n=r s-1, n-k \not \equiv-1(\bmod r)$. Switching $k$ and $n-k$, we get $n-k \not \equiv 0$ $(\bmod r)$ and $k \not \equiv-1(\bmod r)$. This proves the claim.

Claim 2. If there exists a Lehman matrix $A$ with $G_{A}=P_{k} \sqcup P_{n-k}$, then $A$ is unique up to isomorphism.

We continue the discussion from the proof of Claim 1. We previously concluded that

$$
[k+1, k+r-j) \subset \operatorname{row}_{k+1}(A) .
$$

Suppose $k+r-j \in \operatorname{row}_{k+1}(A)$. Then $\sigma(r-j)=k+r-j$ by (11) and (15), so

$$
k+r-j \in \operatorname{col}_{k+r-j}(B)
$$

by (12). Since $k+r-j=r(\ell+1)$, we also have $k+r-j \in \operatorname{row}_{1}(B)$ by (10). Thus,

$$
\operatorname{col}_{k+r-j}(B) \supset\{1, k+r-j\} .
$$

If $i \in \operatorname{row}_{k+1}(A)$ for $i \in[j]$, then $\sigma(r-i)=i$ and

$$
\operatorname{col}_{i}(A)=[i] \cup[k+1, k+r-i],
$$

which contains $\{1, k+r-j\}$, giving a contradiction. Therefore, $\operatorname{row}_{k+1}(A) \cap[j]=\emptyset$, and $\sigma(r-i)=k+r-i$ for $i \in[j]$. This implies

$$
[k+1, k+r) \subset \operatorname{row}_{k+1}(A)
$$

which is another contradiction because rows $k$ and $k+1$ are not adjacent. Hence $k+r-j \notin$ $\operatorname{row}_{k+1}(A)$, so $\sigma(r-j)=j$.

Observe that $j \in \operatorname{row}_{k}(B)$ by (10) since $k=r \ell+j$. Thus, (12) implies that

$$
\operatorname{col}_{j}(B) \supset\{k, k+r-j\} .
$$

If $k+r-i \in \operatorname{row}_{k+1}(A)$ for $i \in[j]$, then $\sigma(r-i)=k+r-i$ and

$$
\operatorname{col}_{k+r-i}(A)=(k-i, k+r-i]
$$


which contains $\{k, k+r-j\}$, giving a contradiction. We conclude that

$$
[k+r-j, k+r) \cap \operatorname{row}_{k+1}(A)=\emptyset .
$$

Now by (15), we conclude that

$$
\begin{gathered}
{[j] \cup[k+1, k+r-j) \subset \operatorname{row}_{k+1}(A) \text { and }} \\
\sigma(i)= \begin{cases}k+i & \text { if } i \in[r-j-1] \\
r-i & \text { if } i \in[r-j, r)\end{cases}
\end{gathered}
$$

We further deduce from (13) and (14) that

$$
\sigma(n-k+i)=\left\{\begin{array}{ll}
k+r-i & \text { if } i \in[j] \\
i & \text { if } i \in[j+1, r)
\end{array} .\right.
$$

We have thus determined the columns $[k+r-1]$ in $A$. This determines $A$ up to a choice of $\sigma([r, n-k])$. Since all $1 \mathrm{~s}$ in rows $[k]$ of $A$ are contained in columns $[k+r-1]$, we can permute the rest of the columns without affecting the first $k$ rows. Therefore, we have shown that if a thin Lehman matrix $A$ exists with $G_{A}=P_{k} \sqcup P_{n-k}$, then $A$ is unique up to isomorphism $\left(P_{k}\right.$ is a path of length $\left.k\right)$.

$(\Longleftarrow)$ Let $r<k<n-r$ and $k=r \ell+j$ for $0<j<r / 2$. We will construct an $n \times n$ $r$-regular thin Lehman matrix $A$ such that $G_{A}=P_{k} \sqcup P_{n-k}$. First we divide $C_{n}^{r}$ and $D_{n}^{s}$ into blocks. Write

$$
C_{n}^{r}=\left[\begin{array}{cc}
A_{11} & 0 \\
A_{21} & A_{22}
\end{array}\right] \quad \text { and } \quad D_{n}^{s}=\left[\begin{array}{ll}
B_{11} & B_{12} \\
B_{21} & B_{22}
\end{array}\right]
$$

where $A_{11}$ and $B_{11}$ are $k \times(k+r-1)$ matrices, and $A_{22}$ and $B_{22}$ are $(n-k) \times(n-k-r+1)$ matrices. Let $P$ be the $(k+r-1) \times(k+r-1)$ permutation matrix such that $A_{21} P$ switches columns

$$
i \leftrightarrow k+r-i \quad \text { for } \quad i \notin(r t+j, r(t+1)), t \in[0, \ell]
$$

and keeps all other columns fixed. Let $Q$ be the $(n-k-r+1) \times(n-k-r+1)$ permutation matrix such that $B_{12} Q$ switches columns

$$
r t-j+i \leftrightarrow r t-i+1 \quad \text { for } \quad i \in[j], t \in[1, s-\ell-2]
$$

and keeps all other columns fixed.

Note that if $\ell=s-2$, then $n-k-r+1=r(s-\ell-1)-j=r-j$, so $Q$ is the identity matrix. If $\ell=s-3$, then $(r-j, r]=(r(s-\ell-2)-j, r(s-\ell-2)]$, so $Q$ only switches one block of columns.

Define $A$ and $B$ by

$$
A=\left[\begin{array}{cc}
A_{11} & 0 \\
A_{21} P & A_{22}
\end{array}\right] \quad \text { and } \quad B=\left[\begin{array}{cc}
B_{11} & B_{12} Q \\
B_{21} P & B_{22}
\end{array}\right] .
$$


Observe that $A$ is $r$-regular with rows $[k]$ and $(k, n]$ forming two disjoint paths in $G_{A}$. To avoid excessive technical details, we leave it to the reader to confirm that $A B^{T}=J+I$, i.e., $A$ and $B$ form a thin Lehman pair. This proves the theorem.

Example. We give an example of $A$ from Theorem 5.1 with $G_{A}=P_{k} \sqcup P_{n-k}$. We also provide the dual $B$. In the example $r=5, s=5, n=24$, and $k=7$.
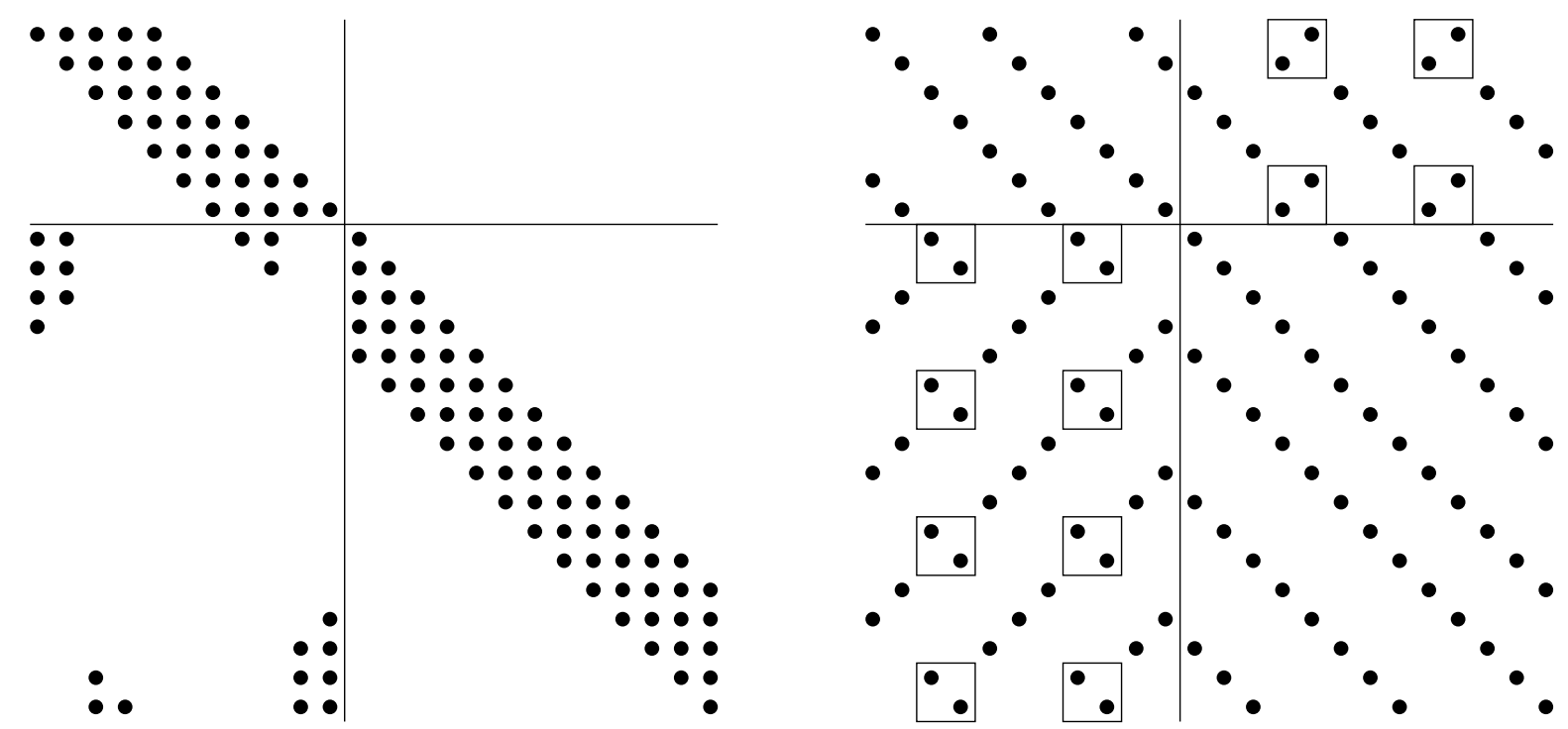

\section{Circulant matrices}

Let $S \subset \mathbb{Z} / n \mathbb{Z}$. We define the circulant matrix $C_{S}$ to be the $n \times n$ matrix with columns indexed by $\mathbb{Z} / n \mathbb{Z}$ and rows equal to the incidence vectors of

$$
i+S:=\{i+s \mid s \in S\}
$$

for $i \in \mathbb{Z} / n \mathbb{Z}$. By definition, any circulant matrix can be written in this form. We study when a circulant matrix is also a Lehman matrix. We show that if $C_{S}$ is a Lehman matrix, then either $C_{S} \simeq C_{n}^{r}$ or the Johnson subgraph $G_{C_{S}}$ has no edges. We then provide some general constructions of circulant Lehman matrices $C_{S}$ such that the Johnson subgraph indeed has no edges.

Note that if $C_{S}$ is a Lehman matrix, then its dual must also be circulant, since a translation of $\mathbb{Z} / n \mathbb{Z}$ is a bijection and the dual is unique. If $C_{S}$ is Lehman for $d>1$, then $G_{C_{S}}$ cannot have any edges. We therefore again only consider thin Lehman matrices.

Proposition 6.1. Let $r=|S|$. If $C_{S}$ is a thin Lehman matrix, then either $C_{S} \simeq C_{n}^{r}$ or $G_{C_{S}}$ has no edges.

Proof. Note that since $C_{S}$ is circulant, the graph $G_{C_{S}}$ is vertex transitive (translating the rows in $\mathbb{Z} / n \mathbb{Z}$ is a graph automorphism). Suppose $G_{C_{S}}$ contains an edge. Then there must exist $t<n$ such that

$$
|S \cap(t+S)|=r-1
$$


Let $\{b\}=S \backslash(t+S)$. There must exist $k \geq 0$ such that

$$
b, b+t, \ldots, b+k t \in S \quad \text { but } \quad b+(k+1) t \notin S,
$$

since otherwise $b+n t=b$ contradicts $b \notin t+S$. Therefore, $\{b+(k+1) t\}=(t+S) \backslash S$.

Define

$$
X=S \backslash\{b, b+t, \ldots, b+k t\} .
$$

We claim that $t+X=X$. Take $a \in X$ and suppose $a+t \notin X$. Then either $a+t \notin S$ or $a+t \in\{b, b+t, \ldots, b+k t\}$. If $a+t \notin S$, then $a+t \in(t+S) \backslash S$, so $a+t=b+(k+1) t$. Hence $a=b+k t \notin X$, a contradiction. Now suppose

$$
a+t \in\{b, b+t, \ldots, b+k t\} .
$$

We cannot have $a+t=b$ since $b \notin t+S$. Therefore, $a+t=b+\ell t$ for $\ell \geq 1$, so $a=b+(\ell-1) t \notin X$, which is another contradiction. We conclude that $t+X=X$.

Suppose $k$ is positive. Since $X-t=X+n t-t=X+(n-1) t$, we have $X-t=X$. Therefore,

$$
S-t=\{b-t, b, \ldots, b+(k-1) t\} \cup(X-t)=\{b-t, b, \ldots, b+(k-1) t\} \cup X .
$$

By assumption $b+(k+1) t \notin S$, so $b+(k+1) t \neq b$. Then $b-t \neq b+k t$, and

$$
|S \cap(S-t)|=r-1 \text {. }
$$

Observe that

$$
t+S=\{b+t, b+2 t, \ldots, b+k t, b+(k+1) t\} \cup X .
$$

Since $b \neq b+(k+1) t$, we also have $|S \cap(t+S)|=r-1$. Note that

$$
S \cap(S-t)=\{b, \ldots, b+(k-1) t\} \cup X \quad \text { and } \quad S \cap(t+S)=\{b+t, \ldots, b+k t\} \cup X \text {. }
$$

Therefore, rows $-t, 0, t$ of $C_{S}$ cannot form a clique in $G_{C_{S}}$ because $S \cap(S-t) \neq S \cap(t+S)$, by (6). If $C_{S}$ is not isomorphic to $C_{n}^{r}$, then $G_{C_{S}}$ is a union of clique trees. As $G_{C_{S}}$ is vertex transitive and thus regular, the only possible clique trees are single cliques. Since rows $-t$ and 0 and rows 0 and $t$ are adjacent, but rows $-t$ and $t$ are not, this is a contradiction. Therefore, we must have $C_{S} \simeq C_{n}^{r}$.

Now suppose $k=0$. Then $S=\{b\} \cup X$ and row $t$ is the incidence vector of

$$
t+S=\{b+t\} \cup X .
$$

Let $B$ be the Lehman dual of $C_{S}$. Take some row $i \in \operatorname{col}_{b}(B)$ distinct from 0 or $t$, so $b \in \operatorname{row}_{i}(B)$. Since $\left|S \cap \operatorname{row}_{i}(B)\right|=1,\left|(t+S) \cap \operatorname{row}_{i}(B)\right|=1$, and $S \cap(t+S)=X$, we must have

$$
b+t \in \operatorname{row}_{i}(B) .
$$

Observe that $|X|=r-1 \geq 2$, and pick distinct $a_{1}, a_{2} \in X$. For $j \in\{1,2\}$, we have $\left\{a_{j}, a_{j}+t\right\} \subset X$, so

$$
\left(b-a_{j}\right)+S \supset\{b, b+t\} .
$$

Thus, the distinct rows $b-a_{1}$ and $b-a_{2}$ of $C_{S}$ both intersect row $i$ of $B$ in at least 2 columns, which is a contradiction. In this case, $C_{S}$ cannot be a thin Lehman matrix. 
The previous lemma motivates the question of when a circulant Lehman matrix can have a Johnson subgraph with no edges. We show that for composite $r$ and $s$, there do exist circulant matrices $C_{S}$ that are thin Lehman matrices with edgeless graphs. To simplify our expressions, we use

$$
\operatorname{AP}(a, k, \delta):=\{a, a+\delta, \ldots, a+(k-1) \delta\} \subset \mathbb{Z} / n \mathbb{Z}
$$

to denote arithmetic progressions of length $k$ and difference $\delta$ starting with element $a$.

Given two subsets $X, Y \subset \mathbb{Z} / n \mathbb{Z}$, let

$$
X+Y:=\{x+y \mid x \in X, y \in Y\} .
$$

Proposition 6.2. Suppose $r=r_{1} r_{2}$ and $s=s_{1} s_{2}$ are composite integers, with $r_{i}, s_{i} \geq 2$. Define

$$
\begin{aligned}
S_{A} & =\mathrm{AP}\left(0, r_{1}, 1\right)+\mathrm{AP}\left(0, r_{2}, r_{1} s_{1}\right) \text { and } \\
-S_{B} & =\mathrm{AP}\left(0, s_{1}, r_{1}\right)+\operatorname{AP}\left(0, s_{2}, r s_{1}\right) .
\end{aligned}
$$

Then $C_{S_{A}}, C_{S_{B}}$ form a thin Lehman pair. Moreover $G_{C_{S_{A}}}, G_{C_{S_{B}}}$ are edgeless graphs.

Proof. Let $P$ be the circulant matrix $C_{\{1\}}$ of order $n$, where $\{1\} \subset \mathbb{Z} / n \mathbb{Z}$. Note that $P$ is a permutation matrix. Then we may write

$$
C_{S_{A}}=\sum_{i \in S_{A}} P^{i} \quad \text { and } \quad C_{S_{B}}=\sum_{i \in S_{B}} P^{i} .
$$

Since $P^{T}=P^{-1}$, we have $\left(C_{S_{B}}\right)^{T}=C_{-S_{B}}$. We can thus express

$$
C_{S_{A}}\left(C_{S_{B}}\right)^{T}=\sum_{(i, j) \in S_{A} \times S_{B}} P^{i-j} .
$$

Since $r_{1}<r_{1} s_{1}$ and $r_{1} s_{1}<r s_{1}$, we see that $\left|S_{A}\right|=r_{1} r_{2}=r$ and $\left|S_{B}\right|=s_{1} s_{2}=s$. Observe that $0 \in S_{A} \cap S_{B}$ and

$$
\left(r_{1}-1\right)+\left(r_{2}-1\right) r_{1} s_{1}=n-\left(s_{1}-1\right) r_{1}-\left(s_{2}-1\right) r s_{1} \in S_{A} \cap S_{B} .
$$

Thus, there are two pairs $(i, j) \in S_{A} \times S_{B}$ with $i-j=0$. It is easy to check that

$$
S_{A}-S_{B}=\mathbb{Z} / n \mathbb{Z} .
$$

Since $n=r s-1$, we conclude that

$$
C_{S_{A}}\left(C_{S_{B}}\right)^{T}=P^{0}+\sum_{i \in \mathbb{Z} / n \mathbb{Z}} P^{i}=J+I .
$$

Since $r_{2} \geq 2$ and $r_{1}+r_{2}\left(r_{1} s_{1}\right)<n$, we can deduce that $G_{C_{S_{A}}}$ has no edges. Then $\omega\left(G_{C_{S_{A}}}\right)=0$ implies $\omega\left(G_{C_{S_{B}}^{T}}\right)=0$ by Lemma 4.1. Since $C_{S_{B}}^{T}=C_{-S_{B}}$, this implies $G_{C_{S_{B}}}$ also has no edges. 
Note that some of the matrices $C_{S_{A}}$ given above may be isomorphic for different choices of $r_{1}, r_{2}, s_{1}, s_{2}$. We believe, however, that the previously mentioned matrices are the only possible circulant thin Lehman matrices, up to isomorphism.

Conjecture 6.3. If $C_{S}$ is a thin Lehman matrix, then $C_{S}$ is isomorphic to $C_{n}^{r}$ or one of the matrices in Proposition 6.2.

We used a computer program to confirm that the conjecture is true when $n<48$.

\section{Open problems}

The classification of Lehman matrices, or even only thin Lehman matrices, is still an open problem. We have used the Johnson subgraph to give structural results for thin Lehman matrices, which we believe will make it easier to enumerate them. We showed that a connected component of the graph uniquely determines the corresponding rows in the Lehman matrix, and we completely classified matrices where their graphs have two connected components. Lehman matrices with graphs containing more connected components have not been classified, however. Some of the constraints we provide for the Johnson subgraph may also be improved upon.

If two matrices are isomorphic, then their Johnson subgraphs are also isomorphic. We noted that the converse is false. It would be highly useful to find some simple structure associated to a Lehman matrix such that the matrix is uniquely determined up to isomorphism. One possibility may be to use colored edges to combine the graphs $G_{A}$ and $G_{B}$ into a single graph. Another possibility is to investigate when row intersections have a fixed size other than $r-1$.

We showed that a circulant Lehman matrix must either be isomorphic to $C_{n}^{r}$ or have an edgeless Johnson graph. We propose a conjecture on the classification of all circulant thin Lehman matrices. In particular, it would be interesting to prove that if $r$ or $s$ is prime, then any $n \times n$ circulant thin Lehman matrix is isomorphic to $C_{n}^{r}$, for $n=r s-1$.

The only known infinite families of Lehman matrices are the thin Lehman matrices and the point-line incidence matrices of nondegenerate finite projective planes. We would like to know if there are any other infinite families of Lehman matrices.

\section{Acknowledgments}

This research was done at the University of Minnesota Duluth with the financial support of the National Science Foundation and the Department of Defense (grant number DMS 0754106) and the National Security Agency (grant number H98230-06-1-0013). The author would like to thank Nathan Pflueger, Aaron Pixton, Nathan Kaplan, Ricky Liu, and Yi Sun for their helpful ideas and suggestions during the research and paper writing processes. I especially thank Joe Gallian for suggesting the research topic and running the University of Minnesota Duluth summer research program. I also thank Yann Kieffer for providing me with computer programs to generate extensive supplies of Lehman matrices. 
I would like to thank Brendan McKay for making his graph automorphism program nauty available online.

\section{References}

[1] W. G. Bridges and H. J. Ryser. Combinatorial designs and related systems. J. Algebra, 13:432-446, 1969.

[2] G. Cornuéjols. Combinatorial optimization, volume 74 of CBMS-NSF Regional Conference Series in Applied Mathematics. Society for Industrial and Applied Mathematics (SIAM), Philadelphia, PA, 2001.

[3] G. Cornuéjols, B. Guenin, and L. Tunçel. Lehman matrices. J. Combin. Theory Ser. $B, 99(3): 531-556,2009$.

[4] D. R. Hughes and F. C. Piper. Projective planes. Springer-Verlag, New York, 1973. Graduate Texts in Mathematics, Vol. 6.

[5] A. Lehman. On the width-length inequality. Math. Programming, 16(2):245-259, 1979.

[6] A. Lehman. The width-length inequality and degenerate projective planes. In Polyhedral combinatorics (Morristown, NJ, 1989), volume 1 of DIMACS Ser. Discrete Math. Theoret. Comput. Sci., pages 101-105. Amer. Math. Soc., Providence, RI, 1990.

[7] C. Lütolf and F. Margot. A catalog of minimally nonideal matrices. Math. Methods Oper. Res., 47(2):221-241, 1998.

[8] J. Wang. A new infinite family of minimally nonideal matrices. J. Combin. Theory Ser. A, 118(2):365-372, 2011. 